\title{
Exercise Training Prevent Decrease of Muscle Strength in Otsuka Long- Evans Tokushima Fatty Rats: A Preliminary Study
}

Toru Tamaki ${ }^{*}$ Masako Ikutomo, Satomi Arakawa, Junya Komagata, Masatoshi Niwa and Ken Muramatsu

${ }^{1}$ Department of Physical Therapy, Health Science University, Yamanashi, Japan

${ }^{2}$ Department of Occupational Therapy, Kyorin University, Tokyo, Japan

\begin{abstract}
Background: Decrease of muscle strength around knee and ankle are considered to contribute several movement disorders in diabetic patient such as an increased risk of falling, altered gait and balance, and increased body sway. In this study, we examine the effect of exercise training on skeletal muscle of type 2 diabetic models.

Methods: Otsuka Long-Evans Tokushima fatty (OLEFT) rats, type 2 diabetic models, were divided into an exercise training group (OLETF-EX, $\mathrm{n}=3$ ) and a sedentary group (OLETF-SED, $\mathrm{n}=3$ ), while LongEvans Tokushima Otsuka (LETO) rats were used as a control group (LETO-SED, $n=4)$. The OLETF-EX rats were trained on a treadmill five times a week for 15 weeks. Data were subjected to two-tailed multiple t-tests with Bonferroni correction following an ANOVA.

Results: The muscle force per body weight was significantly lower tension in MG muscle of OLETFSED animals than LETO-SED animals. On the other hand, twitch tensions of OLETF-EX animals were preserved.

Conclusion: These data suggest that exercise training prevent decrease of muscle strength in type 2 diabetic model.
\end{abstract}

\section{Introduction}

It is well known that both type 1 and type 2 diabetic patients shows decrease of muscle strength around knee and ankle [1,2]. These impaired motor functions may contribute to several movement disorders in diabetic patient such as an increased risk of falling, altered gait and balance, and increased body sway [3-5]. These disturbances, however, can be improved by exercise training [6]. Animal experiments using type 1 diabetic model also showed that decrease of muscle strength predominantly occurred on type II (fast) muscle fibers [7] Interestingly, in contrast to human, endurance-exercise training does not prevent or increase muscle weakness in type 1 diabetic rats [8]. This discrepancy may cause by difference of type of diabetes. In previous study, most of clinical research were conducted on type 2 diabetic patients, on the other hand, animal experiment were conducted on type 1 diabetic model. Therefore, there is a need to examine alterations of muscle strength and effect of exercise using type 2 diabetic models.

The Otuska Long-Evans Tokushima Fatty (OLETF) rat is an established model of human type 2 diabetes mellitus which develops a diabetic syndrome by 25 weeks of age [9]. In this study, we examined the effect of exercise training on medial gastrocnemius (MG) muscle and soleus (SOL) muscle strength in OLETF rats. Because of type dependent muscle atrophy in diabetic model, it is important to compare "fast" MG muscle (predominance of type II fibers) and "slow" SOL muscle (predominance of type Ifibers) [10].

\section{Methods}

All animal experiments were carried out in accordance with the National Institutes of Health guide for the care and use of Laboratory animals (NIH Publications No. 8023, revised 1978). The Animal Ethics Committee of Health Science University approved all the experimental procedures. Data are presented as mean \pm S.D. The significance of differences was evaluated by two-tailed multiple t-tests with Bonferroni correction following an ANOVA using computer software (Prism, GraphPad, La Jolla, CA).

\section{Publication History:}

Received: September 13, 2016

Accepted: October 21, 2016

Published: October 24, 2016

\section{Keywords:}

Diabetes; Diabetic neuropathy; Muscle strength; Exercise training

\section{Experimental Design}

The OLEFT rats were randomly divided into either an exercise training (OLETF-EX, $\mathrm{n}=3$ ) or sedentary group (OLETF-SED, $\mathrm{n}=3$ ), while the LETO rats were used as a control group (LETO-SED, $\mathrm{n}=$ 4). The OLETF rats in the exercise-training group were forced to run on treadmill over the 15 weeks period. In contrast, the OLETF rats in the non-exercise group and the LETO rats were kept without exercise training. Forty-eight hours after the last session of the exercise training, an oral glucose tolerance test (OGTT) was employed to test glucose tolerance. After that, the rats were used to measure muscle contraction.

\section{Exercise Protocol}

From 25 weeks old, the OLETF rats in the exercise group were forced to run on a treadmill for $60 \mathrm{~min}$ once a day, five times a week for 15 weeks. The exercise intensity consisted of running at a speed of $12.0-17.0 \mathrm{~m} / \mathrm{min}$. The initial speed of the treadmill was set at 12 $\mathrm{m} / \mathrm{min}$ and was increased gradually, after 4 weeks from beginning of exercise treadmill speed was reached about $17.0 \mathrm{~m} / \mathrm{min}$.

\section{OGTT}

At 40 weeks old, OGTT was conducted for assay glucose tolerance. After a 12 hours fasting period, animals were anesthetized with $4 \%$ halothane at induction of anesthesia and maintained with inhalation

"Corresponding Author: Dr. Toru Tamaki Department of Physical Therapy, Health Science University, 7187 Kodachi, Fujikawaguchiko, Yamanashi 4010380, Japan, Tel: +81 55583 5298; E-mail: toru-tamaki@kenkoudai.ac.jp

Citation: Tamaki T, Ikutomo M, Arakawa S, Komagata J, Niwa M, et al. (2016) Exercise Training Prevent Decrease of Muscle Strength in Otsuka Long-Evans Tokushima Fatty Rats: A Preliminary Study. Int J Phys Ther Rehab 2: 121. doi: https://doi.org/10.15344/2455-7498/2016/121

Copyright: (C) 2016 Jigami et al. This is an open-access article distributed under the terms of the Creative Commons Attribution License, which permits unrestricted use, distribution, and reproduction in any medium, provided the original author and source are credited. 
Citation: Tamaki T, Ikutomo M, Arakawa S, Komagata J, Niwa M, et al. (2016) Exercise Training Prevent Decrease of Muscle Strength in Otsuka Long-Evans Tokushima Fatty Rats: A Preliminary Study. Int J Phys Ther Rehab 2: 121. doi: https://doi.org/10.15344/2455-7498/2016/121

Page 2 of 3

halothane at 1.5 to $2 \%$. The glucose solution dissolved in water (including $1 \mathrm{~g} / \mathrm{kg}$ glucose) was either injected into the stomach by a polyethylene tube (20-gauge, $40 \mathrm{~mm}$ long curved), and the serum blood glucose level was obtained by pricking the tail vein using glucometer at time $0,30,60,90$ and 120 minutes.

\section{Measurement of Muscle Contraction}

After OGTT, the animals were anesthetized with $4 \%$ halothane at induction of anesthesia and maintained with inhalation halothane at 1.5 to $2 \%$. Subsequently, tibial nerve was severed at a proximal point approximately $2 \mathrm{~cm}$ from the tibial nerve and the nerve was then placed on cuff electrodes. Nerve branches from the tibial nerve, innervating other muscles than the GM / SOL, were also severed. A screw at the tibia fixed the hind limb and the achilles tendon was attached to a force transducer. During the experiment, both the muscle and its surrounding were kept moist at physiological temperature $\left(34-35^{\circ} \mathrm{C}\right)$. The twitch force of MG / SOL was produced by single-pulse nerve supramaximal stimulation at optimum length, defined as the length at which maximal twitch tension is produced. The tensions were digitized with a sample rate of $200 \mathrm{kHz}$ (PowerLab, AD Instruments, Castle Hill, NSW, Australia). After the experiment, maximal force, time to peak tension (TTP) and half-relaxation time (HRT) were calculated from the sampled data.

\section{Results}

\section{OGTT and body weight}

The results of the OGTT for all groups are shown in Table 1 and Figure 1. There was a significant difference between OLETF-SED and LETO-SED at the initial point of the test. On the other hand, there was no difference between OLETF-EX and LETO-SED. At 15 min after the glucose injection, the blood glucose level in OLETFSED was significantly higher than that of OLETF-EX and LETO-SED. Additionally, at $30 \mathrm{~min}$ after the glucose injection, the blood glucose level in OLETF-EX was significantly higher than that LETO-SED. There is significantly higher body weight in both OLETF-SED and OLETF-EX animals compare to LETO-SED animals. On the other hand, OLETF-SED animals showed higher body weight than in LETO-EX(Table 1).

\begin{tabular}{|c|c|c|c|}
\hline & $\begin{array}{l}\text { LETO-SED } \\
(\mathrm{n}=4)\end{array}$ & $\begin{array}{l}\text { OLETF-SED } \\
(n=3)\end{array}$ & $\begin{array}{l}\text { OLETF-EX } \\
(n=3)\end{array}$ \\
\hline $\begin{array}{l}\text { Body weight }(\mathrm{g}) \\
\text { OGTT } \\
\text { Blood glucose } \\
\text { (mg/dl) }\end{array}$ & $511.8 \pm 13.9 \dagger$ & $670.3 \pm 30.2^{*} \dagger$ & $574.3 \pm 23.4^{*}$ \\
\hline $0 \mathrm{~min}$ & $127.3 \pm 11.6$ & $172.6 \pm 39.1^{*} \dagger$ & $129.3 \pm 23.1$ \\
\hline $15 \mathrm{~min}$ & $151.2 \pm 19.2$ & $270.0 \pm 55.6^{\star} \dagger$ & $171.6 \pm 34.1$ \\
\hline $30 \mathrm{~min}$ & $176.7 \pm 37.2$ & $371.3 \pm 12.8^{\star} \dagger$ & $217.3 \pm 12.4^{*}$ \\
\hline $60 \mathrm{~min}$ & $156.5 \pm 28.4$ & $417.0 \pm 56.5^{\star} \dagger$ & $249.0 \pm 46.8^{\star}$ \\
\hline $120 \mathrm{~min}$ & $140.5 \pm 8.8$ & $359.7 \pm 24.5^{\star} \dagger$ & $249.0 \pm 42.0^{*}$ \\
\hline $\begin{array}{l}\text { Table } 1 \text {. Body weig } \\
{ }^{*} \mathrm{P}<0.05 \text { vs. LETO } \\
\dagger \mathrm{P}<0.05 \text { vs. OLET }\end{array}$ & $\begin{array}{l}\text { and results of } \\
\text { EX }\end{array}$ & GTT. & \\
\hline
\end{tabular}

\section{Muscle contraction}

Results of twitch measurements for MG and SOL muscles are shown in Table 2. There is no significant difference between each experimental group in twitch tensions. When expressed per body weight, however, significantly lower tension in MG muscle of OLETFSED animals than LETO-SED animals. On the other hand, twitch tensions of OLETF-EX animals were preserved. There were no differences in either group of both muscles in TTP and HRT.

\section{Discussion}

In present study, there is no significant difference in muscle strength between each group by simple comparison of absolute strength of muscle contraction, although glucose tolerance was improved in OLETF-EX animal. However, we have to take difference of body weight into consideration, because heavy body weight requiring a higher power muscle, on the other hand, low body weight requiring a lower power muscle relatively. Therefore, muscle force per body weight may reflect the actual motor function of each animal. As shown in results, muscle force per body weight was significantly lower in MG muscle of OLETF-SED animals than LETO-SED animals.

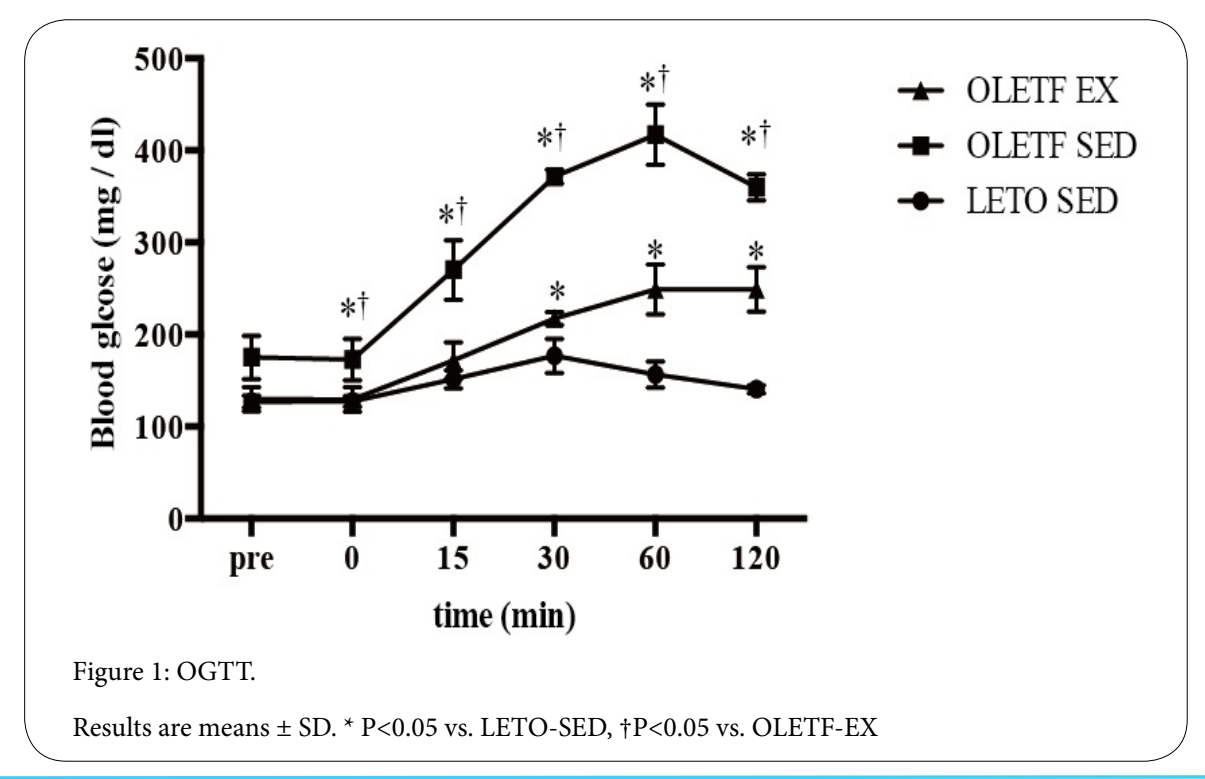


Citation: Tamaki T, Ikutomo M, Arakawa S, Komagata J, Niwa M, et al. (2016) Exercise Training Prevent Decrease of Muscle Strength in Otsuka Long-Evans Tokushima Fatty Rats: A Preliminary Study. Int J Phys Ther Rehab 2: 121. doi: https://doi.org/10.15344/2455-7498/2016/121

Page 3 of 3

\begin{tabular}{|l|l|l|l|}
\hline & $\begin{array}{l}\text { LETO-SED } \\
(\mathbf{n}=4)\end{array}$ & $\begin{array}{l}\text { OLETF-SED } \\
(\mathbf{n}=3)\end{array}$ & $\begin{array}{l}\text { OLETF-EX } \\
(\mathbf{n}=3)\end{array}$ \\
\hline MG muscle & & & \\
\hline Twitch tension & & & $116.6 \pm 2.3$ \\
\hline Maximal force (g) & $102.8 \pm 11.5$ & $105.3 \pm 5.3$ & $0.20 \pm 0.0$ \\
\hline $\begin{array}{l}\text { Muscle force per body } \\
\text { weight (\%) }\end{array}$ & $0.20 \pm 0.0$ & $0.15 \pm 0.0^{\star} \dagger$ & 0.0 \\
\hline Contractile property & & $26.2 \pm 7.0$ & $28.0 \pm 2.0$ \\
\hline TTP (ms) & $25.3 \pm 3.8$ & $15.3 \pm 1.1$ & $18.1 \pm 4.1$ \\
\hline HRT (ms) & $17.4 \pm 6.6$ & & \\
\hline SOL muscle & & & $82.0 \pm 15.5$ \\
\hline Twitch tension & $18.7 \pm 1.9$ & $19.6 \pm 2.7$ & $20.2 \pm 0.4$ \\
\hline Maximal force (g) & $0.04 \pm 0.0$ & $0.03 \pm 0.0$ & $0.03 \pm 0.0$ \\
\hline $\begin{array}{l}\text { Muscle force per body } \\
\text { weight (\%) }\end{array}$ & & & \\
\hline Contractile property & $56.5 \pm 1.1$ & $53.0 \pm 6.5$ & $51.4 \pm 8.4$ \\
\hline TTP (ms) & $55.9 \pm 12.2$ & $72.5 \pm 14.3$ & \\
\hline HRT (ms) & & & \\
\hline
\end{tabular}

Table 2: Contractile properties of MG and SOL muscle.

${ }^{\star} \mathrm{P}<0.05$ vs. LETO-SED

$\dagger \mathrm{P}<0.05$ vs. OLETF-EX

In contrast, that of OLETF-EX animals was preserved. The possibility should be considered however, that preserve of muscle force per body weight was not caused by increased / preserved muscle force but resulted from loss of body weight such as decrease of fat etc. Considering the fact there are no significant difference in muscle force per body weight between LETO-SED and OLETF-EX in spite of OLETF-EX remain significant heavier body weight than in LETO-SED, it's reasonable to consider increased / preserved muscle force has some contribution to preserve of muscle force per body weight. Why only contractile property of MG muscle was altered in diabetic model? This may relate to the fact that decrease of muscle strength predominantly occurred on type II (fast) muscle fibers in diabetic mode [7]. Because, MG muscle fibers are composed of over $90 \%$ type II muscle fibers, the strength of MG muscle may subject to diabetes [10]. Add to this, in accordance with previous study, there is no difference in TTP and HRT of each group [7]. Interestingly, effect of exercise training is exact opposite of previous study that report that endurance-exercise training does not prevent or increase muscle weakness in type 1 diabetic rats [8]. This discrepancy may reflect difference of type of diabetes. Compare to OLETF rats, type 1 diabetic model show much higher blood glucose and show an insulin deficiency and ketoacidosis [7,11]. There is no surprise if such a grave diabetic condition, which is quite different from patients receiving treatment for diabetes, contradicts exercise effect. In fact, exercise training improve motor disturbance in diabetic patients [6]. We think, therefore, OLETF rats i.e., type 2 diabetic model, is much closer to the actual situation of diabetic patients. We should be considered, however, that sample number is too small to conclude exercise training prevent decrease of muscle strength. There is a need for further studies using more large number of sample to clarify the effect of exercise on type 2 diabetic model.

\section{Conclusion}

In summary the present preliminary study has showed possibility that exercise training prevent decrease of muscle strength in type 2 diabetic model. These findings may provide a novel mechanism and treating for deficits in sensorimotor control in diabetic patients.

\section{Competing Interests}

The authors have no competing interests exits.

\section{Author Contributions}

Toru Tamaki: Researched data and wrote the manuscript.

Masako Ikutomo, Satomi Arakawa, Junya Komagata: Researched data. Masatoshi Niwa: Contributed to discussion.

Ken Muramatsu: Contributed to discussion and reviewed/edited manuscript.

\section{Funding}

This work was supported by Research Grant of Health Science University.

\section{References}

1. Andersen H, Nielsen S, Mogensen CE, Jakobsen J (2004) Muscle strength in type 2 diabetes. Diabetes 53: 1543-1548.

2. Andersen H, Poulsen PL, Mogensen CE, Jakobsen J (1996) Isokinetic muscle strength in long-term IDDM patients in relation to diabetic complications. Diabetes 45: 440-445.

3. Andersen H (2012) Motor dysfunction in diabetes. Diabetes/Metabolism Research and Reviews 28: 89-92.

4. Petrofsky J, Lee S, Cuneo ML (2005) Gait characteristics in patients with type 2 diabetes; improvement after administration of rosiglitazone. Med Sci Monit 11: PI43-P151.

5. Uccioli L, Giacomini PG, Monticone G, Magrini A, Durola L, et al. (1995) Body sway in diabetic neuropathy. Diabetes Care 18: 339-344.

6. Allet L, Armand S, de Bie RA, Golay A, Monnin D, et al. (2010) The gait and balance of patients with diabetes can be improved: a randomised controlled trial. Diabetologia 53: 458-466.

7. Cotter M, Cameron NE, Lean DR, Robertson S (1989) Effects of long-term streptozotocin diabetes on the contractile and histochemical properties of rat muscles. Q J Exp Physiol 74: 65-74.

8. Sanchez OA, Snow LM, Lowe DA, Serfass RC, Thompson LV (2005) Effects of endurance exercise-training on single-fiber contractile properties of insulin-treated streptozotocin-induced diabetic rats. J Appl Physiol(1985) 99: 472-478.

9. Kawano K, Hirashima T, Mori S, Saitoh Y, Kurosumi M, et al. (1992) Spontaneous long-term hyperglycemic rat with diabetic complications. Otsuka Long-Evans Tokushima Fatty (OLETF) strain. Diabetes 41: 14221428.

10. Armstrong RB, Phelps RO (1984) Muscle fiber type composition of the rat hindlimb. Am J Anat 171: 259-272.

11. Mansford KR, Opie L (1968) Comparison of metabolic abnormalities in diabetes mellitus induced by streptozotocin or by alloxan. Lancet 1: 670671. 